\title{
Elliptic curves with exceptionally large analytic order of the Tate-Shafarevich groups
}

\author{
Andrzej Dąbrowski and Lucjan Szymaszkiewicz
}

\begin{abstract}
We exhibit 88 examples of rank zero elliptic curves over the rationals with $|\omega(E)|>63408^{2}$, which was the largest previously known value for any explicit curve. Our record is an elliptic curve $E$ with $|ш(E)|=$ $1029212^{2}=2^{4} \cdot 79^{2} \cdot 3257^{2}$. We can use deep results by Kolyvagin, Kato, Skinner-Urban and Skinner to prove that, in some cases, these orders are the true orders of $ш$. For instance, $410536^{2}$ is the true order of $\amalg(E)$ for $E=E_{4}(21,-233)$ from the table in section 2.3.
\end{abstract}

Key words: elliptic curves, Tate-Shafarevich group, central $L$-values

2010 Mathematics Subject Classification: 11G05, 11G40, 11Y50

\section{Introduction}

Let $E$ be an elliptic curve defined over $\mathbb{Q}$ of conductor $N_{E}$, and let $L(E, s)$ denote its $L$-series. Let $\Psi(E)$ be the Tate-Shafarevich group of $E, E(\mathbb{Q})$ the group of rational points, and $R(E)$ the regulator, with respect to the Néron-Tate height pairing. Finally, let $\Omega_{E}$ be the least positive real period of the Néron differential of a global minimal Weierstrass equation for $E$, and define $C_{\infty}(E)=\Omega_{E}$ or $2 \Omega_{E}$ according as $E(\mathbb{R})$ is connected or not, and let $C_{\text {fin }}(E)$ denote the product of the Tamagawa factors of $E$ at the bad primes. The Euler product defining $L(E, s)$ converges for $\operatorname{Re} s>3 / 2$. The modularity conjecture, proven by Wiles-Taylor-Diamond-Breuil-Conrad, implies that $L(E, s)$ has an analytic continuation to an entire function. The Birch and Swinnerton-Dyer conjecture relates the arithmetic data of $E$ to the behaviour of $L(E, s)$ at $s=1$.

Conjecture 1 (Birch and Swinnerton-Dyer) (i) L-function $L(E, s)$ has a zero of order $r=\operatorname{rank} E(\mathbb{Q})$ at $s=1$, 
(ii) $ш(E)$ is finite, and

$$
\lim _{s \rightarrow 1} \frac{L(E, s)}{(s-1)^{r}}=\frac{C_{\infty}(E) C_{\text {fin }}(E) R(E)|\omega(E)|}{\left|E(\mathbb{Q})_{\text {tors }}\right|^{2}} .
$$

If $\boldsymbol{\Psi}(E)$ is finite, the work of Cassels and Tate shows that its order must be a square.

The first general result in the direction of this conjecture was proven for elliptic curves $E$ with complex multiplication by Coates and Wiles in 1976 [6], who showed that if $L(E, 1) \neq 0$, then the group $E(\mathbb{Q})$ is finite. Gross and Zagier [16] showed that if $L(E, s)$ has a first-order zero at $s=1$, then $E$ has a rational point of infinite order. Rubin 28] proves that if $E$ has complex multiplication and $L(E, 1) \neq 0$, then $\omega(E)$ is finite. Let $g_{E}$ be the rank of $E(\mathbb{Q})$ and let $r_{E}$ the order of the zero of $L(E, s)$ at $s=1$. Then Kolyvagin [18] proved that, if $r_{E} \leq 1$, then $r_{E}=g_{E}$ and $\boldsymbol{W}(E)$ is finite. Very recently, Bhargava, Skinner and Zhang [1] proved that at least $66.48 \%$ of all elliptic curves over $\mathbb{Q}$, when ordered by height, satisfy the weak form of the Birch and Swinnerton-Dyer conjecture, and have finite Tate-Shafarevich group.

When $E$ has complex multiplication by the ring of integers of an imaginary quadratic field $K$ and $L(E, 1)$ is non-zero, the $p$-part of the Birch and Swinnerton-Dyer conjecture has been established by Rubin [29] for all primes $p$ which do not divide the order of the group of roots of unity of $K$. Coates et al. [5] 44, and Gonzalez-Avilés [13] showed that there is a large class of explicit quadratic twists of $X_{0}(49)$ whose complex $L$-series does not vanish at $s=1$, and for which the full Birch and Swinnerton-Dyer conjecture is valid (covering the case $p=2$ when $K=\mathbb{Q}(\sqrt{-7})$ ). The deep results by Kato, Skinner and Urban ([32], Theorem 2) and Skinner ([31], Theorem C) allow, in specific cases (still assuming $L(E, 1)$ is non-zero), to establish $p$ part of the Birch and Swinnerton-Dyer conjecture for elliptic curves without complex multiplication for all odd primes $p$.

It has long been known that the order of $\Psi(E)[p]$ can be arbitrarily large for elliptic curves $E$ defined over $\mathbb{Q}$ and $p=2,3$ (for $p=3$, the result is due to Cassels ([3]), and for $p=2$ it is due to McGuinness ([24]). It was later extended for $p=5$ by Fisher [12], and for $p=7$ and 13 by Matsuno [21], but no similar result is known for $p=11$ or $p>13$. Let us mention, that they all used the fact that there are infinitely many elliptic curves defined over $\mathbb{Q}$ with rational $p$-isogenies. We also stress that it has not yet been proven that there exist elliptic curves $E$ defined over $\mathbb{Q}$ for which $\amalg(E)[p]$ is non-zero for arbitrarily large primes $p$.

In our earlier papers, we have investigated (see [11], [7], [8], [9], [10]) some numerical examples of $E$ defined over $\mathbb{Q}$ for which $L(E, 1)$ is non-zero and the order of $\amalg(E)$ is large. 
We extend these numerical results here in this paper, with the largest proved example of $\amalg(E)$ having order $410536^{2}=2^{6} \cdot 7^{2} \cdot 7331^{2}$. We exhibit 88 examples of rank zero elliptic curves over the rationals with $|\omega(E)|>63408^{2}$, which was the largest previously known value for any explicit curve. For some of these examples we can use deep results by Kolyvagin, Kato, Skinner-Urban and Skinner, to prove that these orders are the true orders of $ш$.

Our idea was to use the family $E_{i}(n, p)$ from [11] (see section 2.1 below), within the bounds $20 \leq n \leq 24$ and $0<|p| \leq 5000$ (the calculations in [11] were focused on the pairs $(n, p)$ within the bounds $3 \leq n \leq 19$ and $0<|p| \leq 1000)$. First step was to find good candidates, i.e. the curves $E_{i}(n, p)$ with rank zero and $\max _{i}\left|\Psi\left(E_{i}(n, p)\right)\right|>50000^{2}$. The next step was to calculate $\left|\Psi\left(E_{i}(n, p)\right)\right|$ exactly for all these good candidates. In these steps, we computed (or estimated) the analytic orders of $ய(E)$, using the approximations to $L(E, 1)$. The computations were performed using the computer package PARI/GP [27]. The total running time for the various computational parts was about 9 months.

This research was supported in part by PLGrid Infrastructure. Our computations were carried out in 2016 on the Prometheus supercomputer via PLGrid infrastructure. We also used the HPC cluster HAL9000 and desktop computers Core(TM) 2 Quad Q8300 4GB/8GB, all located at the Department of Mathematics and Physics of Szczecin University.

\section{Results}

The previously largest value for $|ш(E)|$ was $63408^{2}$, found by Dąbrowski and Wodzicki [11]. In ([7], section 5) we propose a candidate with $|ш(E)|>$ $100000^{2}$. Below we present the results of our recent search for elliptic curves with exceptionally large analytic order of the Tate-Shafarevich groups. We exibit 88 examples of rank zero elliptic curves with $|\omega(E)|>63408^{2}$. Our record is an elliptic curve $E=E_{2}(23,-348)$ with $|\omega(E)|=1029212^{2}$. Also note that the prime 19861 divides the orders of $\amalg\left(E_{i}(22,304)\right)$ - the largest (at the moment) prime dividing the order of $\amalg(E)$ of an elliptic curve over $\mathbb{Q}$.

\section{$2.1 \quad$ Preliminaries}

In this section we compute the analytic order of $\amalg(E)$, i.e., the quantity

$$
|ш(E)|=\frac{L(E, 1) \cdot\left|E(\mathbb{Q})_{\text {tors }}\right|^{2}}{C_{\infty}(E) C_{\text {fin }}(E)},
$$


for certain special curves of rank zero. We use the following approximation of $L(E, 1)$

$$
S_{m}=2 \sum_{n=1}^{m} \frac{a_{n}}{n} e^{-\frac{2 \pi n}{\sqrt{N}}},
$$

which, for

$$
m \geq \frac{\sqrt{N}}{2 \pi}\left(2 \log 2+k \log 10-\log \left(1-e^{-2 \pi / \sqrt{N}}\right)\right)
$$

differs from $L(E, 1)$ by less than $10^{-k}$.

Consider (as in [11]) the family

$$
E_{1}(n, p): \quad y^{2}=x(x+p)\left(x+p-4 \cdot 3^{2 n+1}\right)
$$

with $(n, p) \in \mathbb{N} \times(\mathbb{Z} \backslash\{0\})$. Any member of the family admits three isogenous $($ over $\mathbb{Q})$ curves $E_{i}(n, p)(i=2,3,4)$ :

$$
\begin{array}{ll}
E_{2}(n, p): & y^{2}=x^{3}+4\left(2 \cdot 3^{2 n+1}-p\right) x^{2}+16 \cdot 3^{4 n+2} x, \\
E_{3}(n, p): & y^{2}=x^{3}+2\left(4 \cdot 3^{2 n+1}+p\right) x^{2}+\left(4 \cdot 3^{2 n+1}-p\right)^{2} x, \\
E_{4}(n, p): & y^{2}=x^{3}+2\left(p-8 \cdot 3^{2 n+1}\right) x^{2}+p^{2} x
\end{array}
$$

In our calculations, we focused on the pairs of integers $(n, p)$ within the bounds $20 \leq n \leq 24$ and $0<|p| \leq 5000$. Recall that the calculations in [11] were focused on the pairs $(n, p)$ within the bounds $3 \leq n \leq 19$ and $0<|p| \leq 1000$.

The conductors, $L$-series and ranks of isogenous curves coincide, what may differ is the orders of $E(\mathbb{Q})_{\text {tors }}$ and $\Psi(E)$, the real period $\Omega_{E}$, and the Tamagawa number $C_{\text {fin }}(E)$. In our situation we are dealing with 2-isogenies, thus the analytic order of $\amalg(E)$ can only change by a power of 2 .

Notation. Let $N(n, p)$ denote the conductor of the curve $E_{i}(n, p)$. We put $\left|\uplus_{i}\right|=\left|ш\left(E_{i}\right)\right|$.

\subsection{Elliptic curves $E_{i}(n, p)$ with $50000^{2} \leq \max \left(\left|\uplus_{i}\right|\right)<$ $250000^{2}$}

\begin{tabular}{|r|r|r|r|r|r|}
\hline$(n, p)$ & $N(n, p)$ & $\left|W_{1}\right|$ & $\left|Ш_{2}\right|$ & $\left|Ш_{3}\right|$ & $\left|Ш_{4}\right|$ \\
\hline$(20,-756)$ & 42551829106699251024 & $27993^{2}$ & $55986^{2}$ & $27993^{2}$ & $27993^{2}$ \\
$(20,-2000)$ & 190293894141760627320 & $15081^{2}$ & $60324^{2}$ & $15081^{2}$ & $60324^{2}$ \\
$(20,192)$ & 109418989131512359065 & $3780^{2}$ & $60480^{2}$ & $945^{2}$ & $60480^{2}$ \\
\hline \multicolumn{5}{|c|}{ Continued on next page } \\
\hline
\end{tabular}




\begin{tabular}{|c|c|c|c|c|c|}
\hline$(n, p)$ & $N(n, p)$ & $\left|W_{1}\right|$ & $\left|\uplus_{2}\right|$ & $\left|\uplus_{3}\right|$ & $\left|\uplus_{4}\right|$ \\
\hline$\overline{(22,-692)}$ & 11978814802342833513168 & $15194^{2}$ & $30388^{2}$ & $7597^{2}$ & $60776^{2}$ \\
\hline$(21,-128)$ & 1969541804367222465954 & $34234^{2}$ & $68468^{2}$ & $34234^{2}$ & $68468^{2}$ \\
\hline$(20,-180)$ & 60788327295284644080 & $20970^{2}$ & $41940^{2}$ & $10485^{2}$ & $83880^{2}$ \\
\hline$(21,3)$ & 31512668869875559452120 & $10962^{2}$ & $43848^{2}$ & $5481^{2}$ & $87696^{2}$ \\
\hline$(20,-2448)$ & 1653442502431742344680 & $22028^{2}$ & $88112^{2}$ & $22028^{2}$ & $88112^{2}$ \\
\hline$(20,2704)$ & 11379574869677285146824 & $48538^{2}$ & $97076^{2}$ & $97076^{2}$ & $48538^{2}$ \\
\hline$(21,12)$ & 281363114909603209392 & $12768^{2}$ & $102144^{2}$ & $3192^{2}$ & $102144^{2}$ \\
\hline$(20,-608)$ & 16631686347989878669080 & $25787^{2}$ & $103148^{2}$ & $51574^{2}$ & $51574^{2}$ \\
\hline$(21,192)$ & 984770902183611232737 & $54648^{2}$ & $109296^{2}$ & $27324^{2}$ & $109296^{2}$ \\
\hline$(20,4788)$ & 25871512096873143639456 & $27745^{2}$ & $110980^{2}$ & $27745^{2}$ & $27745^{2}$ \\
\hline$(20,2680)$ & 23938195173261478962720 & $14474^{2}$ & $115792^{2}$ & $14474^{2}$ & $57896^{2}$ \\
\hline$(20,-801)$ & 34625031227394133415352 & $29338^{2}$ & $58676^{2}$ & $29338^{2}$ & $117352^{2}$ \\
\hline$(22,1344)$ & 62040566837567507664447 & $60930^{2}$ & $121860^{2}$ & $30465^{2}$ & $60930^{2}$ \\
\hline$(20,-1436)$ & 1832369310703810488288 & $32455^{2}$ & $129820^{2}$ & $32455^{2}$ & $129820^{2}$ \\
\hline$(20,4768)$ & 10032879618827902147272 & $16254^{2}$ & $130032^{2}$ & $8127^{2}$ & $65016^{2}$ \\
\hline$(21,-24)$ & 31512668869875559452768 & $34092^{2}$ & $68184^{2}$ & $17046^{2}$ & $136368^{2}$ \\
\hline$(20,-1376)$ & 37640132261240251922904 & $70010^{2}$ & $140020^{2}$ & $140020^{2}$ & $70010^{2}$ \\
\hline$(22,64)$ & 8862938119652501095881 & $72306^{2}$ & $144612^{2}$ & $36153^{2}$ & $144612^{2}$ \\
\hline$(21,-1536)$ & 1969541804367222468066 & $75897^{2}$ & $151794^{2}$ & $75897^{2}$ & $151794^{2}$ \\
\hline$(20,-6)$ & 14005630608833581979328 & $19248^{2}$ & $76992^{2}$ & $4812^{2}$ & $153984^{2}$ \\
\hline$(22,304)$ & 27493195799738370745848 & $39722^{2}$ & $158888^{2}$ & $19861^{2}$ & $79444^{2}$ \\
\hline$(21,1516)$ & 11663380372737145525968 & $25866^{2}$ & $206928^{2}$ & $12933^{2}$ & $103464^{2}$ \\
\hline$(21,480)$ & 39390836087344449300840 & $54110^{2}$ & $216440^{2}$ & $27055^{2}$ & $108220^{2}$ \\
\hline$(23,1452)$ & 6451697601805864768272 & $55698^{2}$ & $222792^{2}$ & $27849^{2}$ & $222792^{2}$ \\
\hline
\end{tabular}

2.3 Elliptic curves $E_{i}(n, p)$ with $\max \left(\left|\uplus_{i}\right|\right) \geq 250000^{2}$

\begin{tabular}{|r|r|r|r|r|r|}
\hline$(n, p)$ & $N(n, p)$ & \multicolumn{1}{|c|}{$\left|{ }_{1}\right|$} & \multicolumn{1}{|c|}{$\left|W_{2}\right|$} & \multicolumn{1}{|c|}{$\left|W_{3}\right|$} & \multicolumn{1}{|c|}{$\left|W_{4}\right|$} \\
\hline$(21,4)$ & 15756334434937779726048 & $130614^{2}$ & $261228^{2}$ & $65307^{2}$ & $261228^{2}$ \\
$(21,1248)$ & 102416173827095568122280 & $70375^{2}$ & $281500^{2}$ & $70375^{2}$ & $70375^{2}$ \\
$(20,-201)$ & 234594312697962498467304 & $141540^{2}$ & $141540^{2}$ & $283080^{2}$ & $141540^{2}$ \\
$(23,960)$ & 398832215384362549313205 & $96254^{2}$ & $385016^{2}$ & $48127^{2}$ & $192508^{2}$ \\
$(24,832)$ & 373306953599763346160205 & $75780^{2}$ & $303120^{2}$ & $37890^{2}$ & $151560^{2}$ \\
$(20,1120)$ & 30637316956823460343320 & $20440^{2}$ & $327040^{2}$ & $20440^{2}$ & $81760^{2}$ \\
$(23,-84)$ & 17448909423065861532624 & $184991^{2}$ & $369982^{2}$ & $184991^{2}$ & $184991^{2}$ \\
$(22,480)$ & 354517524786100043822760 & $99938^{2}$ & $399752^{2}$ & $49969^{2}$ & $199876^{2}$ \\
$(23,-8)$ & 7441767284139709375008 & $102120^{2}$ & $204240^{2}$ & $51060^{2}$ & $408480^{2}$ \\
$(21,-233)$ & 149845956054714394972728 & $51317^{2}$ & $205268^{2}$ & $51317^{2}$ & $410536^{2}$ \\
$(23,-96)$ & 638131544614980078907464 & $264696^{2}$ & $529392^{2}$ & $132348^{2}$ & $529392^{2}$ \\
$(24,-96)$ & 302272836922885300534872 & $412146^{2}$ & $824292^{2}$ & $206073^{2}$ & $824292^{2}$ \\
$(23,-348)$ & 37011629587668844576720608 & $514606^{2}$ & $1029212^{2}$ & $257303^{2}$ & $1029212^{2}$ \\
\hline \hline
\end{tabular}




\subsection{Birch and Swinnerton-Dyer conjecture for elliptic curves with exceptionally large analytic order of Tate-Shafarevich groups}

In this subsection, we will use the deep results by Kato, Skinner-Urban and Skinner, to prove the full version of the Birch-Swinnerton-Dyer conjecture for some elliptic curves $E_{i}(n, p)$ with exceptionally large analytic order of Tate-Shafarevich groups.

Let $\bar{\rho}_{E, p}: \operatorname{Gal}(\overline{\mathbb{Q}} / \mathbb{Q}) \rightarrow \mathrm{GL}_{2}\left(\mathbb{F}_{p}\right)$ denote the Galois representation on the $p$-torsion of $E$. Assume $p \geq 3$. As mentioned in [14, the work of Kato [17] together with the works of Mazur and Rubin [23], and Matsuno [20], implies the following result.

Theorem 2 Let $E$ be an optimal elliptic curve over $\mathbb{Q}$ with conductor $N_{E}$. Assume that $p \backslash\left\langle 6 N_{E}\right.$, and $\bar{\rho}_{E, p}$ is surjective. If $L(E, 1) \neq 0$, then $\amalg(E)$ is finite and

$$
\operatorname{ord}_{p}(|\Psi(E)|) \leq \operatorname{ord}_{p}\left(\frac{L(E, 1)}{C_{\infty}(E)}\right) .
$$

For elliptic curves without complex multiplication, the following theorem of Skinner-Urban and Skinner implies that the $p$-part of the Birch and Swinnerton-Dyer conjecture holds for nice primes $p$ in the rank zero case.

Theorem 3 ([32], Theorem 2; [31], Theorem C) Let E be an elliptic curve over $\mathbb{Q}$ with conductor $N_{E}$. Suppose: (i) E has good ordinary or multiplicative reduction at $p$; (ii) there exists a prime $q \neq p$ such that $q \| N_{E}$ and $\bar{\rho}_{E, p}$ is ramified at $q$; (iii) $\bar{\rho}_{E, p}$ is surjective. If moreover $L(E, 1) \neq 0$, then the p-part of the Birch and Swinnerton-Dyer conjecture holds true, that is, we have

$$
\operatorname{ord}_{p}(|ш(E)|)=\operatorname{ord}_{p}\left(\frac{\left|E(\mathbb{Q})_{\text {tors }}\right|^{2} L(E, 1)}{C_{\infty}(E) C_{\text {fin }}(E)}\right) .
$$

Remark 4 (i) The condition (ii) can be removed in the good ordinary case by the results of $X$. Wan [33], so we will omit it in the calculations below. (ii) Of course, surjectivity of $\bar{\rho}_{E, p}$ implies its irreducibility.

For the following curves from the tables in 2.2 and 2.3 , we can apply the above results by Kato, Skinner-Urban and Skinner: $E_{i}(22,-692)$, $E_{i}(20,-608), E_{i}(20,-1436), E_{i}(20,4788), E_{i}(22,304), E_{i}(22,64), E_{i}(21,1516)$, $E_{i}(21,4), E_{i}(21,-233)$.

Let us give some details for the curves $E_{i}=E_{i}(20,-1436)$. We can use the above results to show that $\left|\uplus_{1}\right|=5^{2} \times 6491^{2}$ is the true order of $\amalg\left(E_{1}\right)$ (and, hence, all $\left|\uplus_{i}\right|$ are the true orders of $\amalg\left(E_{i}\right)$ ). 
(i) We have $N_{E_{1}}=2^{5} \times 3 \times 7 \times 31 \times 257 \times 359 \times 107323 \times 8883041$.

(ii) We have $j_{E_{1}}=\frac{2^{6} 285451^{3} 4660272567723053424015171049538317^{3}}{3^{82} 7^{8} 31^{2} 257^{2} 359^{2} 107323^{2} 8883041^{2}}$. The representation $\bar{\rho}_{E_{1}, p}$ is surjective for any prime $p \geq 19$ by Prop. 1.8 in [35]. On the other hand, Prop. 6.1 in [35] gives a criterion to determine whether $\bar{\rho}_{E, p}$ is surjective or not for any non-CM elliptic curve $E$ and any prime $p \leq 11$. For instance, the representation $\bar{\rho}_{E, 5}$ is not surjective if and only if $j_{E}=\frac{5^{3}(t+1)(2 t+1)^{3}\left(2 t^{2}-3 t+3\right)^{3}}{\left(t^{2}+t-1\right)^{5}}$ or $j_{E}=\frac{5^{2}\left(t^{2}+10 t+5\right)^{3}}{t^{5}}$ or $j_{E}=t^{3}\left(t^{2}+5 t+40\right)$ for some $t \in \mathbb{Q}$. We have checked, using Pari/GP, impossibility of these three cases to hold. For the primes $p=13$ and $p=17$ (actually for any $p \geq 5$ ), we can give a less computational proof of surjectivity as follows (suggested by the referee). First, it is known due to B. Mazur (see Theorem 3 in [22] or Theorem 1.3 in [25]), that for any elliptic curve $E$ over $\mathbb{Q}$ with all its 2-division points defined over $\mathbb{Q}$, the representation $\bar{\rho}_{E, p}$ is absolutely irreducible for any prime $p \geq 5$. Using the irreducibility, and the fact that the denominator of $j_{E_{1}}$ is not a $p$ th power, we can apply Lemmas 1 and 2 in Chapter IV, Section 3.2 of [30].

(iii) $E_{1}$ has good ordinary reduction at 5 and 6491: $\left(N_{E_{1}}, 5\right)=\left(N_{E_{1}}, 6491\right)$ $=1$, and $a_{5}\left(E_{1}\right)=2, a_{6491}\left(E_{1}\right)=108$.

(iv) $E_{1}$ has multiplicative reduction at any $p \in\{3,7,31,257,359,107323$, $8883041\}$. Take $q=7$. Then $7 \| N_{E_{1}}$, and $\bar{\rho}_{E_{1}, p}$ is ramified at 7 for these $p \neq 7$, since these $p$ 's do not divide $\operatorname{ord}_{7}\left(\Delta_{E_{1}}\right)$. Take $q=31$. Then $31 \| N_{E_{1}}$, and $\bar{\rho}_{E_{1}, 7}$ is ramified at 31 , since 7 do not divide $\operatorname{ord}_{31}\left(\Delta_{E_{1}}\right)$.

(v) Using Magma [2] we see that $Ш\left(E_{1}\right)[2]$ is trivial, and it is easy to conclude that the order of $ш\left(E_{1}\right)$ is odd.

Remark. The curves $E=E_{i}(23,-348)$ have additive reduction at 3, and the above results by Kolyvagin, Kato, Skinner-Urban and Skinner are not enough to prove that the analytic orders of $\amalg$ are the true ones in these cases. These methods only show that, say, the true order of $ய\left(E_{2}(23,-348)\right)$ is $1029212^{2} \cdot 3^{2 k}=2^{4} \cdot 3^{2 k} \cdot 79^{2} \cdot 3257^{2}$, for some non-negative integer $k$. To have $k=0$, we additionally need to prove that $\amalg(E)[3]$ is trivial. One way is to use Magma [2] to show that $\operatorname{Sel}(E)[3]=0$. We had run ThreeSelmerGroup(E) subroutine for about 2,5 months, but without success, and we stopped the calculations. A second way is to use a result of Gross (see [15], Prop. 2.1, Prop. 2.3 or [19], Theorem 0.5) and some Magma computations. Let $y_{K} \in$ $E(K)$ be the basic Heegner point attached to $E$ and a suitable imaginary quadratic field $K$. If $y_{K} \notin 3 E(K)$, then we obtain $\omega(E / K)\left[3^{\infty}\right]=0$ as desired. But we didn't manage to compute $y_{K}$, since all our attempts required recomputing $L(E, 1)$. 


\subsection{Values of the Goldfeld-Szpiro ratio}

Let

$$
G S(E):=\frac{|ш(E)|}{\sqrt{N_{E}}}
$$

denote the Goldfeld-Szpiro ratio of an elliptic curve E. Eleven examples of elliptic curves with $G S(E) \geq 1$ are given by de Weger [34], the largest value being $6.893 \ldots$. Another forty-seven examples with $G S(E) \geq 1$ are produced by Nitaj [26], his largest value of $G S(E)$ being $42.265 \ldots$. For all of these examples the conductor does not exceed $10^{10}$. The article of Dąbrowski and Wodzicki [1] produce two examples with $G S(E) \geq 1$ for curves with much larger conductors.

The largest values of $G S(E)$ that we observed for our curves are given below. The notation $E_{i, j}(n, p)$ means that the given values of $|\omega(E)|$ and $G S(E)$ are shared by the isogeneous curves $E_{i}(n, p)$ and $E_{j}(n, p)$.

\begin{tabular}{|r|r|c|}
\hline$E$ & $|W(E)|$ & $G S(E)$ \\
\hline$E_{4}(23,-8)$ & $408480^{2}$ & $1.9342096803 \ldots$ \\
$E_{2,4}(24,96)$ & $824292^{2}$ & $1.2358410273 \ldots$ \\
$E_{2}(23,-84)$ & $369982^{2}$ & $1.0362798350 \ldots$ \\
$E_{4}(20,-180)$ & $83880^{2}$ & $0.9024159172 \ldots$ \\
$E_{2,4}(21,12)$ & $102144^{2}$ & $0.6220025144 \ldots$ \\
$E_{2,4}(23,1452)$ & $222792^{2}$ & $0.6179625870 \ldots$ \\
$E_{2}(20,1120)$ & $327040^{2}$ & $0.6110494864 \ldots$ \\
$E_{2,4}(21,4)$ & $261228^{2}$ & $0.5436405656 \ldots$ \\
$E_{2,4}(21,-1536)$ & $151794^{2}$ & $0.5191903468 \ldots$ \\
\hline \hline
\end{tabular}

Note that our record elliptic curves $E=E_{2,4}(23,-348)$ have relatively small Goldfeld-Szpiro ratio: $G S(E)=0.1741167606 \ldots$.

Acknowledgement. We thank an anonymous referee for the constructive criticism and comments which improved the final version.

\section{References}

[1] M. Bhargava, Ch. Skinner, W. Zhang, A majority of elliptic curves over $\mathbb{Q}$ satisfy the Birch and Swinnerton-Dyer conjecture, arxiv.org/abs/1407.1826 
[2] W. Bosma, J. Cannon, C. Playoust, The Magma Algebra System I. The user language, J. Symbolic Comput. 24 (1997), no. 3-4, 235-265

[3] J.W.S. Cassels, Arithmetic on curves of genus 1. VI. The TateSafarevic group can be arbitrarily large, J. reine angew. Math. 214/215 (1964), 65-70

[4] J. Coates, Lectures on the Birch-Swinnerton-Dyer Conjecture, Notices of the ICCM 1 (2013), 29-46

[5] J. Coates, Y. Li, Y. Tian, S. Zhai, Quadratic twists of elliptic curves, Proc. London Math. Soc. 110 (2015), 357-394

[6] J. Coates, A. Wiles, On the conjecture of Birch and Swinnerton-Dyer, Invent. Math. 39 (1977), 223-251

[7] A. Dąbrowski, T. Jędrzejak, L. Szymaszkiewicz, Behaviour of the order of Tate-Shafarevich groups for the quadratic twists of $X_{0}(49)$, In: Elliptic Curves, Modular Forms and Iwasawa Theory (in honour of John Coates' 70th birthday), Springer Proceedings in Mathematics and Statistics 188 (2016), 125-158

[8] A. Dąbrowski, L. Szymaszkiewicz, Orders of TateShafarevich groups for the quadratic twists of elliptic curves, arXiv:1611.07840v1[math.NT]

[9] A. Dąbrowski, L. Szymaszkiewicz, Orders of Tate-Shafarevich groups for the Neumann-Setzer type elliptic curves, Math. Comput. 87 (2018), 1509-1522

[10] A. Dąbrowski, L Szymaszkiewicz, Orders of Tate-Shafarevich groups for the cubic twists of $X_{0}(27)$, Banach Center Publications 118 (2019), $125-135$

[11] A. Dąbrowski, M. Wodzicki, Elliptic curves with large analytic order of $ய(E)$, In: Algebra, Arithmetic and Geometry (in honour of Yu.I. Manin, vol. I), Progress in Math. 269 (2009), 407-421

[12] T. Fisher, Some examples of 5 and 7 descent for elliptic curves over $\mathbb{Q}$, Journal of the European Mathematical Society 3(2) (2001), 169-201

[13] C.D. Gonzalez-Avilés, On the conjecture of Birch and Swinnerton-Dyer, Trans. Amer. Math. Soc. 349 (1997), 4181-4200 
[14] G. Grigorov, A. Jorza, S. Patrikis, W.A. Stein, C. Tarnita, Computational verification of the Birch and Swinnerton-Dyer conjecture for individual elliptic curves, Math. Comput. 78 (2009), 2397-2425

[15] B. Gross, Kolyvagin's work on modular elliptic curves. In: L-functions and arithmetic (Durham, 1989), London Mathematical Society Lecture Note Series, vol. 153, Cambridge University Press, 1989, p. 235-256

[16] B. Gross, D. Zagier, Heegner points and derivatives of L-series, Invent. Math. 84 (1986), 225-320

[17] K. Kato, p-adic Hodge theory and values of zeta functions of modular forms, In: Cohomologies p-adiques et applications arithmétiques, III, (edited by P. Berthelot et al), Astérisque 295, 117-290, Société Mathématique de France, Paris, 2004

[18] V. Kolyvagin, Finiteness of $E(\mathbb{Q})$ and $\boldsymbol{\omega}(E)$ for a class of Weil curves, Math. USSR Izv. 32 (1989), 523-541

[19] A. Matar, J. Nekovár̆, Kolyvagin's result on the vanishing of $\mathrm{W}(E / K)\left[p^{\infty}\right]$ and its consequences for anticyclotomic Iwasawa theory, J. Théory des Nombres de Bordeaux 31 (2019), 455-501

[20] K. Matsuno, Finite $\Lambda$-submodules of Selmer groups of abelian varieties over cyclotomic $\mathbb{Z}_{p}$-extensions, J. Number Theory 99 (2003), 415-443

[21] K. Matsuno, Construction of elliptic curves with large Iwasawa lambdainvariants and large Tate-Shafarevich groups, Manuscripta Math. 122 (2007), 289-304

[22] B. Mazur, Rational isogenies of prime degree, Invent. Math. 44 (1978), 129-162

[23] B. Mazur, K. Rubin, Kolyvagin systems. Mem. Amer. Math. Soc. 168(799) (2004)

[24] F. McGuinness, The Cassels pairing in a family of elliptic curves, $\mathrm{PhD}$ thesis, Brown University, 1982

[25] L. Merel, Arithmetic of elliptic curves and diophantine equations, J. Théor. Nombres Bordeaux 11 (1999), 173-200

[26] A. Nitaj, Invariants des courbes de Frey-Hellegouarch et grands groupes de Tate-Shafarevich, Acta Arithm. 93 (2000), 303-327 
[27] The PARI Group, PARI/GP version 2.7.2, Bordeaux, 2014, http://pari.math.u-bordeaux.fr/

[28] K. Rubin, Tate-Shafarevich groups and L-functions of elliptic curves with complex multiplication, Invent. Math. 89 (1987), 527-560

[29] K. Rubin, The "main conjectures" of Iwasawa theory for imaginary quadratic fields, Invent. Math. 103 (1991), 25-68

[30] J.-P. Serre, Abelian l-adic representations and elliptic curves, McGill University lecture notes, W. A. Benjamin, Inc., New York-Amsterdam 1968

[31] Ch. Skinner, Multiplicative reduction and the cyclotomic main conjecture for $G L_{2}$, Pacific J. Math. 283 (2016), 171-200

[32] Ch. Skinner, E. Urban, The Iwasawa main conjectures for $G L_{2}$, Invent. Math. 195 (2014), 1-277

[33] X. Wan, The Iwasawa main conjecture for Hilbert modular forms, Forum of Mathematics, Sigma 3 (2015), 95 pages

[34] B.M.M. de Weger, $A+B=C$ and big w's, Quart. J. Math. 49 (1998), $105-128$

[35] D. Zywina, On the surjectivity of mod $l$ representations associated to elliptic curves, arXiv:1508.07661v1 [math.NT] 31 Aug 2015

Institute of Mathematics, University of Szczecin, Wielkopolska 15, 70451 Szczecin, Poland; E-mail addresses: andrzej.dabrowski@usz.edu.pl and dabrowskiandrzej7@gmail.com; lucjansz@gmail.com 